\title{
Nanoscale Compositional Analysis of a Thermally Processed Entropy-Stabilized Oxide via Correlative TEM and APT
}

\author{
David R. Diercks ${ }^{1}$, Geoff Brennecka ${ }^{1}$, Brian P. Gorman ${ }^{1}$, Christina M. Rost ${ }^{2}$ and Jon-Paul Maria ${ }^{3}$ \\ 1. Colorado School of Mines, Department of Metallurgical and Materials Engineering, Golden, CO USA \\ 2. University of Virginia, Department of Mechanical and Aerospace Engineering, Charlottesville, VA \\ USA \\ 3. North Carolina State University, Department of Materials Science and Engineering, Raleigh, NC USA
}

The recent demonstration of entropy-stabilized oxides (ESOs) [1] suggests new avenues for controlling the microstructures and, thus, properties within this new family of oxide ceramics through a combination of composition control and thermal processing. In order to better understand the possibilities for engineering specific properties, a detailed understanding at the atomic scale is desirable. The exploration of ESO microstructure has been largely focused on that of the quenched, single phase material [1,2]. However, study of the multi-phase material that results from controlled cooling of the single phase material can provide insight into the destabilization mechanisms and methods of manipulating the microstructure. The correlated use of transmission electron microscopy (TEM) and atom probe tomography (APT) is well-suited for such analyses [3,4].

Here, the previously presented $\mathrm{Mg}_{0.2} \mathrm{Ni}_{0.2} \mathrm{Co}_{0.2} \mathrm{Cu}_{0.2} \mathrm{Zn}_{0.2} \mathrm{O}$ (E1) sample [1] is explored after air quenching from $1000{ }^{\circ} \mathrm{C}$ to room temperature and after slow cooling in a furnace at $2{ }^{\circ} \mathrm{C} / \mathrm{min}$ to room temperature. APT specimens were prepared from the bulk samples using a modified focused ion beam (FIB) lift-out technique [5] where specimens were mounted on TEM grids in holders compatible with the FIB, TEM, and APT instruments [3]. This allowed for TEM imaging of the specimens both before and after APT analysis as shown in Figure 1.

In the quenched samples, TEM indicates the vast majority of the sample is composed of the rocksalt phase with grains several microns in size, consistent with previous analyses [1]. APT provides further evidence of the random distribution of cation species throughout these grains via nearest neighbor analyses as shown for $\mathrm{Cu}$ in Figure 2. A couple of regions which contain a dense number of grains only tens of nanometers in size were identified. APT analysis indicates that these regions are enriched in Ni, deficient in $\mathrm{Mg}$ and $\mathrm{O}$, and possibly slightly enriched in $\mathrm{Co}, \mathrm{Cu}$, and $\mathrm{Zn}$ relative to the overall composition. It is not currently clear if those regions are representative of the sample-wide distribution.

In the slow-cooled samples, TEM provides direct visual confirmation of the $\mathrm{CuO}$ tenorite phase that was previously identified via $\mathrm{x}$-ray diffraction [1]. This phase has a needle-like structure as shown in Figure 1, and electron diffraction indicates a number of the needles have an orientation relationship with the matrix. Nearest neighbor analyses within the APT data from these specimens indicate that even in the matrix (non-CuO regions) of the slow-cooled material, the $\mathrm{Cu}$ now exhibits a slight clustering tendency as shown in Figure 2b. 


\section{References:}

[1] C. M. Rost et al, Nature communications 6 (2015), p 6.

[2] D. Berardan et al, Journal of Alloys and Compounds (2017) in press.

[3] B. P. Gorman et al, Microscopy Today 16 (2008), p. 42.

[4] A. Devaraj et al, International Materials Reviews (2017) p. 1.

[5] K. Thompson et al, Ultramicroscopy 107 (2007) p. 131.
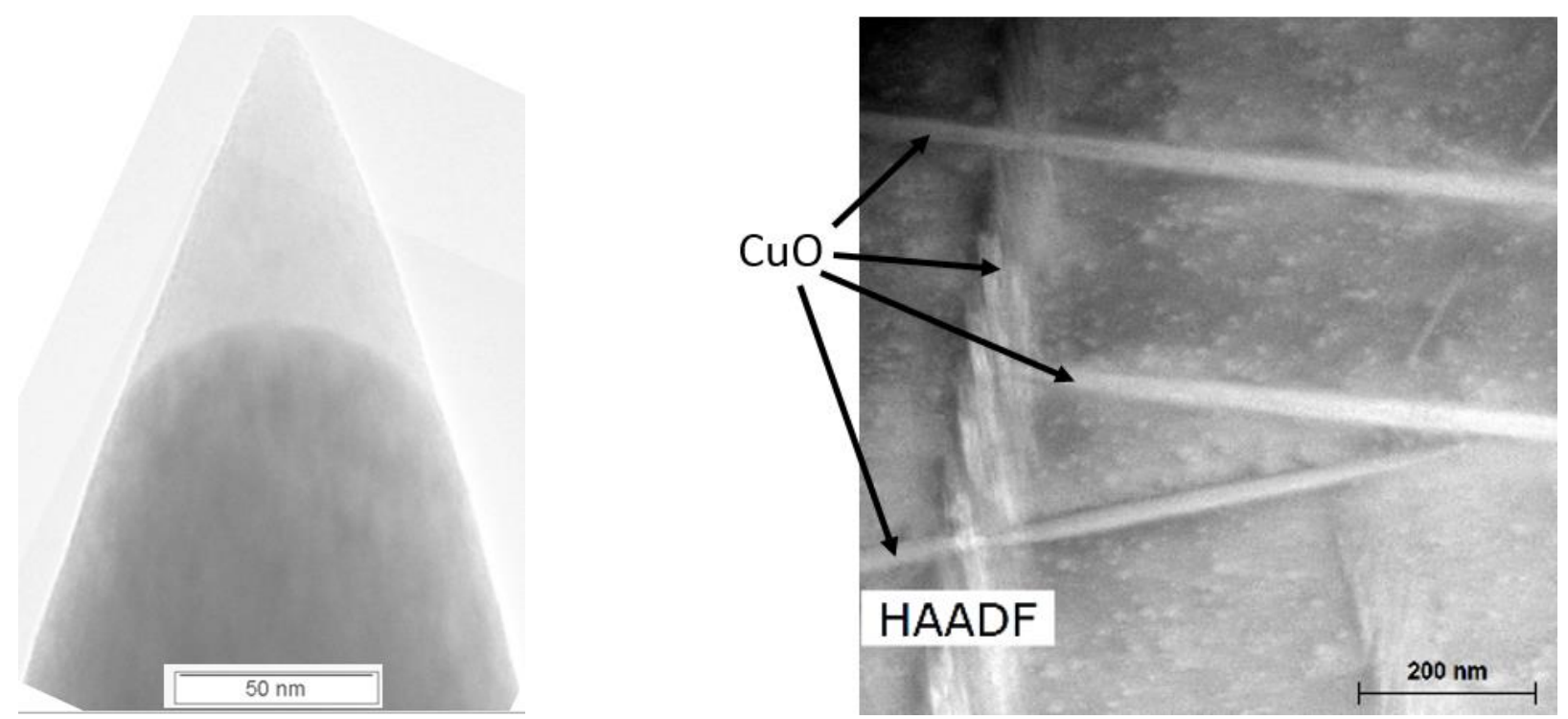

Figure 1. (left) Overlay of the TEM images before and after APT analysis of a slow-cooled specimen containing only the matrix material. (right) Scanning TEM high angle annular dark field (HAADF) image of the slow-cooled material with several $\mathrm{CuO}$ needles indicated.
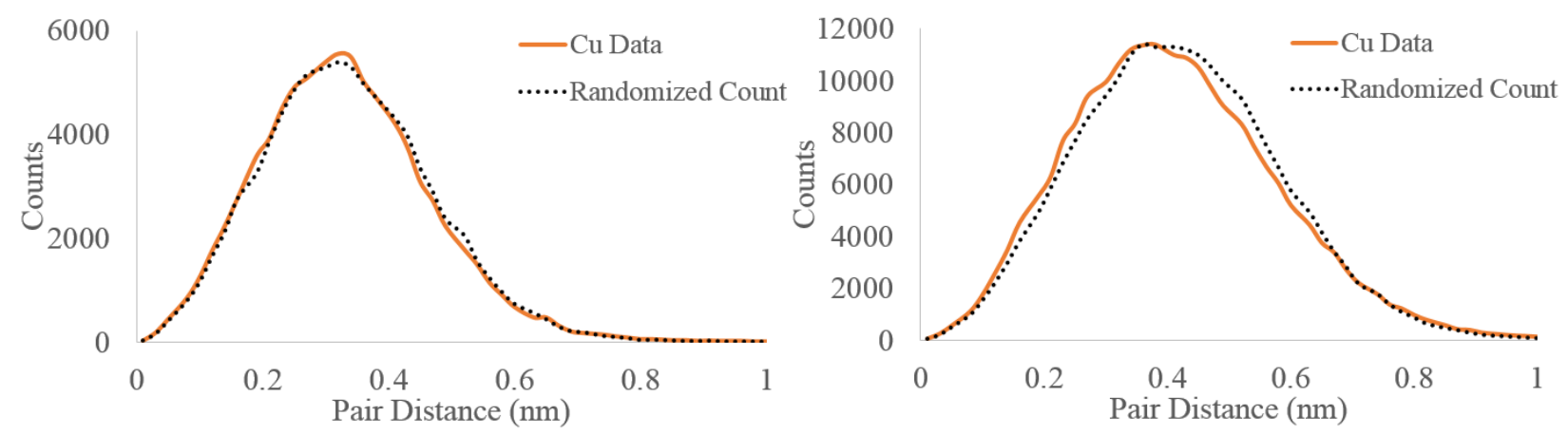

Figure 2. (left) Nearest neighbor distribution of the $\mathrm{Cu}$ species from the APT analysis of a specimen from the quenched sample compared to a random distribution. (right) Nearest neighbor distribution of the $\mathrm{Cu}$ species from the APT analysis of a specimen from the matrix region of a slow-cooled sample compared to a random distribution. 